\title{
MAXIMAL ELEMENTS AND EQUILIBRIA OF GENERALIZED GAMES FOR $U$-MAJORIZED AND CONDENSING CORRESPONDENCES
}

\author{
GEORGE XIAN-ZHI YUAN and E. TARAFDAR
}

(Received 29 November 1993 and in revised form 10 October 1996)

\begin{abstract}
In this paper, we first give an existence theorem of maximal elements for a new type of preference correspondences which are $U$-majorized. Then some existence theorems for compact (resp., non-compact) qualitative games and generalized games in which the constraint or preference correspondences are U-majorized (resp., $\Psi$ condensing) are obtained in locally convex topological vector spaces.
\end{abstract}

Keywords and phrases. $\Psi$-condensing mappings, $\mathcal{U}$ class, $U$-majorized, open lower sections, lower semicontinuous, upper semicontinuous, fixed point, mathematical economics, maximal element, selection theorem, equilibrium point, abstract economy, generalized game.

1991 Mathematics Subject Classification. 47H04, 47H10, 47H11, 47N10, 49J45, 52A07, 54C60, 55M25, 90A14, 90D06, 90D13.

1. Introduction. The existence of equilibrium in an abstract economy with compact strategy sets in $\mathbb{R}^{n}$ was proved in a seminal paper of Debreu [5]. The theorem of Debreu extended the earlier work of Nash in game theory. Since then there have been many generalizations of Debreu 's theorem by Borglin and Keiding [2], Gale and Mas-Colell [12], Florenzano [10], Shafer and Sonnenschein [28], Tan and Yuan [30], Tarafdar [31], Toussaint [33], Tian [32], Tulcea [17], Yannelis and Prabhakar [36] and the references wherein. Following the work of Gale and Mas-Colell [13] and Borglin and Keiding [2] on non-ordered preference relations, many theorems on the existence of maximal elements of preference relations, which may not be transitive or complete, have been proved by Bergstrom [1], Mehta [23], Toussaint [33], Tucela [17], Yannelis [35], Yannelis and Prabhakar [36], and Walker [34]. However, their existence theorems of maximal elements deal with preference correspondences which have lower open sections or are majorized by correspondences with lower open sections.

It is well known that if a correspondence has an open graph, then it has open upper and lower sections and, thus, it must be lower semicontinuous. However, a continuous correspondence need not have open lower or upper sections in general. Also, in the infinite settings, the set of feasible allocations is not necessarily compact in the commodity spaces. The motivations for economists continually to be interested in setting forth conditions for the existence of equilibria come from the importance of generalized games (also called abstract economies) in the study of markets and other general games and from the restrictions of the existing theorems. Since we encounter many kinds of preferences in various economic situations, it is important that we consider several types of preferences and obtain some existence results for such 
correspondences in non-compact and non-paracompact settings.

The objective of this paper is as follows. We first give some existence theorems of maximal elements and equilibria for generalized games and qualitative games in which the preferences are majorized by upper semicontinuous correspondences instead of being majorized by correspondences which have lower open sections (e.g., see Yannelis and Prabhakar [36] and the references wherein). Then some existence theorems of equilibria for non-compact generalized games are, also, given in locally convex topological vector spaces for $\Psi$-condensing correspondences in infinite dimensional locally convex topological vector spaces.

Our intention is to merely illustrate a certain technique that we think will be of use in various problems of mathematical economics. Many other results of the type proved here may be proved under more general conditions.

Let $A$ be a nonempty set. We denote by $2^{A}$ the family of all subsets of $A$. If $A$ is a subset of a topological space $X$, we denote by $\operatorname{cl}_{X}(A)$ the closure of $A$ in $X$. If $A$ is a subset of a vector space, we denote by co $A$ the convex hull of $A$. If $A$ is a nonempty subset of a topological vector space $E$ and $S, T: A \longrightarrow 2^{E}$ are two correspondences, then $\operatorname{co} T, T \cap S: A \longrightarrow 2^{E}$ are correspondences defined by (co) $T(x):=\operatorname{co} T(x)$ and $(T \cap S)(x):=T(x) \cap S(x)$ for each $x \in A$, respectively. If $X$ and $Y$ are topological spaces and $T: X \rightarrow 2^{Y}$ is a correspondence, then

(1) $T$ is said to be upper semicontinuous at $x \in X$ if for any open subset $U$ of $Y$ containing $T(x)$, the set $\{z \in X: T(z) \subset U\}$ is an open neighborhood of $x$ in $X$;

(2) $T$ is upper semicontinuous (on $X$ ) if $T$ is upper semicontinuous at $x$ for each $x \in X$;

(3) the Graph of $T$, denoted by $\operatorname{Graph}(T)$, is the set $\{(x, y) \in X \times Y: y \in T(x)\}$;

(4) the correspondence $\bar{T}: X \rightarrow 2^{Y}$ is defined by $\bar{T}(x)=\left\{y \in Y:(x, y) \in \mathrm{cl}_{X \times Y}\right.$ $\operatorname{Graph}(T)\}$; and

(5) the correspondence $\operatorname{cl} T: X \longrightarrow 2^{Y}$ is defined by $\operatorname{cl} T(x)=\operatorname{cl}_{Y}(T(x))$ for each $x \in X$. It is easy to see that $\operatorname{cl} T(x) \subset \bar{T}(x)$ for each $x \in X$. We remark here that in defining the upper semicontinuity of $T$ at $x \in X$, we do not require that $T(x)$ be nonempty.

Let $X$ and $Y$ be two topological spaces and let $T: X \rightarrow 2^{Y}$ be a correspondence. The mapping $T$ is said to have a maximal element if there exists a point $x \in X$ such that $T(x)=\varnothing$. Let $X$ be a topological space, $Y$ be a nonempty subset of a vector space $E$, $\theta: X \rightarrow E$ be a mapping and $\phi: X \rightarrow 2^{Y}$ be a correspondence. Then

(1) $\phi$ is said to be of class $U_{\theta}$ if

(a) for each $x \in X, \theta(x) \notin \phi(x)$ and

(b) $\phi$ is upper semicontinuous with closed and convex values in $Y$;

(2) $\phi_{x}$ is a $U_{\theta}$-majorant of $\phi$ at $x$ if there is an open neighborhood $N(x)$ of $x$ in $X$ and $\phi_{x}: N(x) \longrightarrow 2^{Y}$ such that

(a) for each $z \in N(x), \phi(z) \subset \phi_{x}(z)$ and $\theta(z) \notin \phi_{x}(z)$ and

(b) $\phi_{x}$ is upper semicontinuous with closed and convex values;

(3) $\phi$ is said to be $\mho_{\theta}$-majorized if for each $x \in X$ with $\phi(x) \neq \varnothing$, there exists a $u_{\theta}$-majorant $\phi_{x}$ of $\phi$ at $x$.

We remark that when $X=Y$ and $\theta=I_{X}$, the identity mapping on $X$, our notions of a $u_{\theta}$-majorant of $\phi$ at $x$ and a $U_{\theta}$-majorized correspondence are generalization of 
upper semicontinuous correspondences which are irreflexive (i.e., $x \notin \phi(x)$ for all $x \in X)$ and have closed convex values.

When

(I) $X=Y$ and is a nonempty convex subset of the topological vector space $E$ and $\theta=I_{X}$, the identity mapping on $X$; or the case

(II) $X=\prod_{i \in I} X_{i}$ and $\theta=\pi_{j}: X \longrightarrow X_{j}$ is the projection of $X$ onto $X_{j}$ and $Y=X_{j}$ is a nonempty convex subset of a topological vector space, we write $U$ in place of $u_{\theta}$.

Let $I$ be a countable or uncountable set of agents. For each $i \in I$, suppose her/his choice or strategy set $X_{i}$ is a nonempty subset of a topological vector space. Let $X=$ $\prod_{i \in I} X_{i}$. For each $i \in I$, let $P_{i}: X \longrightarrow 2^{X_{i}}$ be a correspondence. Following the notion of Gale and Mas-Colell [13], the collection $\Gamma=\left(X_{i}, P_{i}\right)_{i \in I}$ is called a qualitative game. A point $\hat{x} \in X$ is said to be an equilibrium of the game $\Gamma$ if $P_{i}(\hat{x})=\varnothing$ for all $i \in I$. For each $i \in I$, let $A_{i}$ be a subset of $X_{i}$. Then for each fixed $k \in I$, we define $\prod_{j \in I, j \neq k} A_{j} \otimes A_{k}:=$ $\left\{x=\left(x_{i}\right)_{i \in I}: x_{i} \in A_{i}\right.$ for all $\left.i \in I\right\}$.

A generalized game (abstract economy) is a family of quadruples $\Gamma=\left(X_{i} ; A_{i}, B_{i}\right.$; $\left.P_{i}\right)_{i \in I}$, where $I$ is a (finite or infinite) set of players (agents) such that, for each $i \in I, X_{i}$ is a nonempty subset of a topological vector space and $A_{i}, B_{i}: X=\prod_{j \in I} X_{j} \longrightarrow 2^{X_{i}}$ are constraint correspondences and $P_{i}: X \rightarrow 2^{X_{i}}$ is a preference correspondence. When $I=\{1, \ldots, N\}$, where $N$ is a positive integer, $\Gamma=\left(X_{i} ; A_{i}, B_{i} ; P_{i}\right)_{i \in I}$ is, also, called an $N$-person game. An equilibrium of $\Gamma$ is a point $\hat{x} \in X$ such that, for each $i \in I$, $\hat{x}_{i}=\pi_{i}(\hat{x}) \in \overline{B_{i}}(\hat{x})$ and $A_{i}(\hat{x}) \cap P_{i}(\hat{x})=\varnothing$. We remark that when $\overline{B_{i}}(\hat{x})=\operatorname{cl}_{X_{i}} B_{i}(\hat{x})$ (which is the case when $B_{i}$ has a closed graph in $X \times X_{i}$ ); in particular, when $\operatorname{cl} B_{i}$ is upper semicontinuous with closed values, and if $A_{i}=B_{i}$ for each $i \in I$, our definition of an equilibrium point coincides with the standard definition, e.g., see Borglin and Keiding [2], Tulcea [17] and Yannelis and Prabhakar [36].

Throughout this paper, $C$ denotes a lattice with a least element zero. Let $X$ be a Hausdorff locally convex topological vector space. Then (e.g., see Furi and Vignoil [11]) a mapping $\Psi: 2^{X} \rightarrow C$ is called a measure of non-compactness provided that the following conditions hold for any $A, B \in 2^{X}$ :

(1) $\Psi(A)=0$ if and only if $A$ is precompact;

(2) $\Psi(\overline{\mathrm{CO}} A)=\Psi(A)$, where $\overline{\mathrm{co}} A$ denotes the closed convex hull of $A$;

(3) $\Psi(A \cup B)=\max \{\Psi(A), \Psi(B)\}$.

It follows from (3) that if $A \subset B$, then $\Psi(A) \leq \Psi(B)$. The above notion is a generalization of the set-measure of non-compactness of Kuratowski [20] and the ball-measure of non-compactness of Sadovskii [27] defined in terms of either a family of seminorms when $X$ is a locally convex topological vector space or a single norm when $X$ is a Banach space. For more details, we refer the readers to Fitzpatrick and Petryshyn [9] and the references wherein.

Let $\Psi: 2^{X} \rightarrow C$ be a measure of non-compactness of $X$ and $D \subset X$. A mapping $T: D \longrightarrow 2^{X}$ is called $\Psi$-condensing provided that if $\Omega \subset D$ and $\Psi(T(\Omega)) \geq \Psi(\Omega)$, then $\Omega$ is relatively compact.

Note that if $T: D \longrightarrow 2^{X}$ is a compact mapping (i.e., $T(D):=\cup_{x \in D} T(x)$ is precompact), then $T$ is $\Psi$-condensing for any measure of non-compactness $\Psi$. Various $\Psi$-condensing mappings, which are compact or not compact, have been considered 
by Borisovich et al. [3], Furi and Vignoli [11], Gohberg et al. [21], Massatt [22], Nussbaum [24], Reich [26], Petryshyn and Fitzpatrick [25], and others. Moreover, when the measure of non-compactness $\Psi$ is either the set-measure of non-compactness or ball measure of non-compactness, $\Psi$-condensing mappings are called condensing mappings.

2. Some lemmas. Before we give our main results, we first have:

LEMMA 2.1. Let D be a nonempty closed convex subset of a locally convex topological vector space $E$ and $T: D \longrightarrow 2^{D}$ is $\Psi$-condensing, where $\Psi: 2^{E} \longrightarrow C$ is a measure of noncompactness. Then there exists a nonempty compact and convex subset $K$ of $X$ such that $T: K \longrightarrow 2^{K}$.

Proof. Let $x_{0}$ be an element of $D$ and consider the family $\mathscr{F}$ of all closed convex subsets $C$ of $D$ such that $x_{0} \in C$ and $T: C \longrightarrow 2^{C}$. Clearly, $\mathscr{F}$ is nonempty. Let $C_{0}=$ $\cap_{C \in \mathscr{F}} C$. Then $C_{0}$ is a nonempty closed and convex and $x_{0} \in C_{0}$. If $x \in C_{0}, T(x) \subset C$ for all $C$ so that $T: C_{0} \longrightarrow 2^{C_{0}}$.

Now, we prove that $C_{0}$ is a nonempty compact convex subset of $D$. Suppose that $C_{0}$ were not compact. Then since $T$ is $\Psi$-condensing mapping, $\Psi\left(T\left(C_{0}\right)\right) \notin \Psi\left(C_{0}\right)$. Let $C_{1}=\overline{\mathrm{Co}}\left(\left\{x_{0}\right\} \cup T\left(C_{0}\right)\right)$. Then $C_{1} \subset C_{0}$ which implies that $T\left(C_{1}\right) \subset T\left(C_{0}\right) \subset C_{1}$. Hence, $C_{1} \in \mathscr{F}$ and $C_{0} \subset C_{1}$. Therefore, $C_{0}=C_{1}$, a contradiction because $\Psi\left(C_{1}\right)=$ $\Psi\left[\overline{\mathrm{CO}}\left(\left\{x_{0}\right\} \cup T\left(C_{0}\right)\right)\right]=\Psi\left(T\left(C_{0}\right)\right)$, where the second equality holds because of the definition of $\Psi$. This contradiction proves Lemma 2.1.

We, also, need the following result which is Lemma 2.10 in Tan and Yuan [29]:

LEMMA 2.2. Let $X$ and $Y$ be two topological spaces and let $A$ be a closed (resp., open) subset of $X$. Suppose $F_{1}: X \longrightarrow 2^{Y}, F_{2}: A \longrightarrow 2^{Y}$ are lower (resp., upper) semicontinuous such that $F_{2}(x) \subset F_{1}(x)$ for all $x \in A$. Then the mapping $F: X \rightarrow 2^{Y}$ defined by

$$
F(x)= \begin{cases}F_{1}(x), & \text { if } x \notin A ; \\ F_{2}(x), & \text { if } x \in A\end{cases}
$$

is, also, lower (resp., upper) semicontinuous.

The following result is essentially due to Hildenbrand [15, p. 23-24] (see also Klein and Thompson [19, Thm. 7.3.10, p. 86]):

LEMMA 2.3. Let $X$ be a topological space and $Y$ be a normal space. If $F, G: X \rightarrow 2^{Y}$ have closed values and are upper semicontinuous at $x \in X$, then $F \cap G$ is, also, upper semicontinuous at $x$.

Proof. If $F(x) \cap G(x) \neq \varnothing$, the conclusion follows from Hildenbrand [15, Prop. B.III.2, p. 23-23] (see also Klein and Thompson [19, Thm. 7.3.10, p. 86]). If $F(x) \cap G(x)=\varnothing$, it is easy to see that there exists an open neighborhood $X$ of $x$ in $X$ such that $F(z) \cap P(z)=\varnothing$ for all $z \in N$ (since $Y$ is normal) and so $F \cap G$ is, also, upper semicontinuous at $x$.

We remark here that in Lemma 2.3, we do not require $F(x) \cap G(x) \neq \varnothing$ for each $x \in X$. 
3. Maximal element theorems. In order to give our maximal element theorems, we have the following selection result for $U$-majorized correspondences.

THEOREM 3.1. Let $X$ be a paracompact space and let $Y$ be a nonempty normal subset of a topological vector space $E$. Let $\theta: X \rightarrow E$ and $P: X \rightarrow 2^{Y}$ be U-majorized. Then there exists a correspondence $\Psi: X \rightarrow 2^{Y}$, of class $u$ such that $P(x) \subset \Psi(x)$ for each $x \in X$.

Proof. Since $P$ is $U$-majorized, for each $x \in X$ with $P(x) \neq \varnothing$, let $N(x)$ be an open neighborhood of $x$ in $X$ and $\psi_{x}: N(x) \longrightarrow 2^{Y}$ be such that

(1) for each $z \in N(x), P(z) \subset \psi_{x}(z)$ and $\theta(z) \notin \psi_{x}(z)$ and

(2) $\psi_{x}$ is upper semicontinuous with closed and convex values.

Since $X$ is paracompact and $X=\cup_{x \in X} N(x)$, by Dugundji [7, Thm. VIII.1.4, p. 162], the open covering $\{N(x)\}$ of $X$ has an open precise neighborhood-finite refinement $\left\{N^{\prime}(x)\right\}$. For each $x \in X$, define $\psi_{x}^{\prime}: X \longrightarrow 2^{Y}$ by

$$
\psi_{x}^{\prime}(z)= \begin{cases}\psi_{x}(z), & \text { if } z \in N^{\prime}(x) ; \\ Y, & \text { if } z \notin N^{\prime}(x),\end{cases}
$$

then $\psi_{x}^{\prime}$ is, also, upper semicontinuous on $X$ by Lemma 2.2 such that $P(z) \subset \psi_{x}^{\prime}(z)$ for each $z \in X$.

Now, define $\Psi: X \rightarrow 2^{Y}$ by $\Psi(z)=\cap_{x \in X} \psi_{x}^{\prime}(z)$ for each $z \in X$. Clearly, $\Psi$ has closed and convex values and $P(z) \subset \Psi(z)$ for each $z \in X$. Let $z \in X$ be given, then $z \in N^{\prime}(x)$ for some $x \in X$ so that $\psi_{x}^{\prime}(z)=\psi_{x}(z)$ and, hence, $\Psi(z) \subset \psi_{x}(z)$. As $\theta(z) \notin \psi_{x}(z)$, we must, also, have that $\theta(z) \notin \Psi(z)$. Thus, $\theta(z) \notin \Psi(z)$ for all $z \in X$.

Now, we show that $\Psi$ is upper semicontinuous. For any given $u \in X$, there exists an open neighborhood $M_{u}$ of $u$ such that the set $\left\{x \in X: M_{u} \cap N(x) \neq \varnothing\right\}$ is finite, say $\{x(u, 1), \ldots, x(u, n(u))\}$. Thus, we have that

$$
\Psi(w)=\bigcap_{x \in X} \psi_{x}^{\prime}(w)=\overbrace{i=1}^{n(u)} \psi_{x(u, i)}^{\prime}(w) \quad \text { for all } w \in M_{u} .
$$

For $i=1, \ldots, n(u)$, since each $\psi_{x(u, i)}^{\prime}$ is upper semicontinuous on $X$ and, hence, on $M_{u}$ with closed values and since $Y$ is normal, by Lemma 2.3, $\Psi: M_{u} \rightarrow 2^{Y}$ is, also, upper semicontinuous at $u$. Since $M_{u}$ is open, $\Psi: X \longrightarrow 2^{Y}$ is, also, upper semicontinuous at $u$. Hence, $\Psi$ is of class $u$.

Now, we prove the following theorem concerning the existence of a maximal element for $U$-majorized correspondences:

THEOREM 3.2. Let $X$ be a nonempty convex subset of a Hausdorff locally convex topological vector space and let $D$ be a nonempty compact subset of $X$. Let $P: X \longrightarrow$ $2^{D}$ be $U$-majorized (i.e., $\bigcup_{I_{X}}$-majorized). Then there exists a point $x \in \operatorname{co} D$ such that $P(x)=\varnothing$.

Proof. Suppose the contrary, i.e., for all $x \in \operatorname{co} D, P(x) \neq \varnothing$. Then for each $x \in$ $\operatorname{co} D, P(x) \neq \varnothing$ and $\operatorname{co} D$ is, also, paracompact (e.g., see Ding et al. [6, Lem. 2]). Now, applying Theorem 3.1, there exists a correspondence $\Psi: \operatorname{co} D \longrightarrow 2^{D}$ of class $U$ such that for each $x \in \operatorname{co} D, P(x) \subset \Psi(x)$. Since $\Psi$ is upper semicontinuous with nonempty 
closed and convex values, by Himmelberg [16, Thm. 2], there exists $x \in \operatorname{co} D$ such that $x \in \Psi(x)$. This contradicts that $\Psi$ is of class $U$. Hence, the conclusion must hold.

We note that Theorem 3.2 is closely related, though not comparable, to those existence theorems of maximal elements of Bergstrom [1], Gale and Mas-Colell [13], Mehta [23], Yannelis [35], Yannelis and Prabhackar [36], and Walker [34].

4. The existence of equilibria in locally convex spaces. In this section, we prove a new existence theorem of equilibria of a generalized game in which the intersection of constraint and preference correspondences are $U$-majorized and with any (countable or uncountable) set of players in locally convex topological vector spaces.

THEOREM 4.1. Let $\Gamma=\left(X_{i} ; A_{i}, B_{i} ; P_{i}\right)_{i \in I}$ be a generalized game (abstract economy), where I is any (countable or uncountable) set of agents (players) such that for each $i \in I$ :

(i) $X_{i}$ is a nonempty compact and convex subset of a locally Hausdorff topological vector space $E_{i}$;

(ii) for each $x \in X\left(=\prod_{i \in I} X_{i}\right), A_{i}(x)$ is nonempty and $A_{i}(x) \subset \overline{B_{i}}(x)$, where $\overline{B_{i}}(x)$ is convex;

(iii) the set $E^{i}=\left\{x \in X: A_{i}(x) \cap P_{i}(x) \neq \varnothing\right\}$ is open and paracompact in $X$;

(iv) the mapping $A_{i} \cap P_{i}: X \rightarrow 2^{X_{i}}$ is U-majorized on $E^{i}$.

Then $\Gamma$ has an equilibria point, i.e., there exists a point $x \in X$ such that $\pi_{i}(x) \in \overline{B_{i}}(x)$ and $A_{i}(x) \cap P_{i}(x)=\varnothing$ for all $i \in I$.

Proof. First, we note that if $E^{i}=\varnothing$ for all $i \in I$, then the conclusion follows by Fan-Glicksberg fixed point theorem (e.g., see Fan [8] or Glicksberg [14]).

Let $I_{0}=\left\{i \in I: E^{i} \neq \varnothing\right\}$. Without loss of generality, we may assume that $I_{0} \neq \varnothing$.

CASE 1. For each $i \in I_{0}$ by (iv) and Theorem 3.1, there exists a mapping $\psi_{i}: E^{i} \longrightarrow 2^{X_{i}}$ which is upper semicontinuous with closed and convex values and $A_{i}(x) \cap P_{i}(x) \subset$ $\psi_{i}(x)$ for each $x \in E^{i}$. Since $\overline{B_{i}}: X \rightarrow 2^{X_{i}}$ is, also, upper semicontinuous with closed and convex values, the mapping $\psi_{i} \cap \overline{B_{i}}: X \rightarrow 2^{X_{i}}$ is, also, upper semicontinuous with nonempty closed and convex values by Lemma 2.3 on $E^{i}$. Define a correspondence $\phi_{i}: X \longrightarrow 2^{X_{i}}$ by

$$
\phi_{i}(x)= \begin{cases}\overline{B_{i}}(x), & \text { if } x \notin E^{i}, \\ \left(\psi_{i} \cap \overline{B_{i}}\right)(x), & \text { if } x \in E^{i} .\end{cases}
$$

Then Lemma 2.2 implies that $\phi_{i}$ is upper semicontinuous with nonempty closed and convex values.

CASE 2. For $i \in I \backslash I_{0}$, we define a correspondence $\phi: X \longrightarrow 2^{X_{i}}$ by $\phi_{i}:=\overline{B_{i}}(x)$ for each $x \in X$. Then $\phi$ is upper semicontinuous with nonempty compact and convex values.

Finally, we define a correspondence $\Psi: X \rightarrow 2^{X}$ by $\Psi(x):=\prod_{i \in I} \phi_{i}(x)$. Then $\Psi$ is also upper semicontinuous with nonempty compact and convex values. Fan-Glicksberg fixed point theorem implies that there exists a point $x \in X$ such that $x \in \Psi(x)$. If there exists $i \in I_{0}$ such that $x \in E^{i}$, then $\pi_{i}(x) \in \phi_{i}(x)=\overline{B_{i}}(x) \cap \psi_{i}(x) \subset$ $\psi_{i}(x)$ which contradicts that $\psi_{i}$ is $U$-majorized on $E^{i}$. Therefore, $x \notin E^{i}$ for all $i \in I_{0}$, 
i.e., there exists an $i^{\prime} \notin I_{0}$ such that $x \in E^{i^{\prime}}$. By the definition of $\Psi$, we must have $\pi_{i}(x) \in \overline{B_{i}}(x)$ and $A_{i}(x) \cap P_{i}(x)=\varnothing$ for all $i \in I$.

By Theorem 4.1, we have the following existence theorem of equilibria for a qualitative game:

THEOREM 4.2. Let $\Gamma=\left(X_{i}, P_{i}\right)_{i \in I}$ be a qualitative game such that for each $i \in I$,

(a) $X_{i}$ is a nonempty compact and convex subset of a Hausdorff locally convex topological vector space $E_{i}$;

(b) the set $E^{i}=\left\{x \in X: P_{i}(x) \neq \varnothing\right\}$ is open and paracompact in $X$; and

(c) $P_{i}$ is U-majorized on $E^{i}$.

Then there exists a point $x \in X$ such that $P_{i}\left(x_{i}\right)=\varnothing$ for all $i \in I$.

Proof. For each $i \in I$, let $A_{i}, B_{i}: X \rightarrow 2^{X_{i}}$ be defined by $A_{i}(x)=B_{i}(x)=X_{i}$ for each $x \in X$, then the generalized game $\Gamma=\left(X_{i} ; A_{i}, B_{i} ; P_{i}\right)_{i \in I}$ satisfy all hypotheses of Theorem 4.1. Therefore, the conclusion of Theorem 4.2 follows.

It seems natural to replace the condition (iii) of Theorem 4.1 by the condition that "the set $E^{i}=\left\{x \in X: A_{i}(x) \cap P_{i}(x) \neq \varnothing\right\}$ is closed" for each $i \in I$, however, the following simple example shows that this cannot be done.

EXAMPLe A. Let $I=\{1\}$ and $X=[0,1]$. Define $A, P: X \rightarrow 2^{X}$ by

$$
A(x)= \begin{cases}{[1 / 2,1],} & \text { if } x \in[0,1 / 2), \\ {[0,1],} & \text { if } x=1 / 2, \\ {[0,1 / 2],} & \text { if } x \in(1 / 2,1]\end{cases}
$$

and

$$
P(x)= \begin{cases}x / 4, & \text { if } x \in[1 / 2,1], \\ \varnothing, & \text { if } x \in[0,1 / 2) .\end{cases}
$$

It is easy to see that $A$ and $P$ are both upper semicontinuous with closed and convex values and $x \notin P(x)$ for each $x \in X$, so that $A \cap P$ is $U$-majorized. We, also, know that the subset $E=\{x \in X: A(x) \cap P(x) \neq \varnothing\}=[1 / 2,1]$ is closed in [0,1] and $A, P$ satisfy all hypotheses of Theorem 4.1 except the condition (iii). But the unique fixed point $1 / 2$ of the correspondence $A$ is such that $A(1 / 2) \cap P(1 / 2)=[0,1] \cap\{1 / 8\} \neq \varnothing$. Thus, the generalized game $([0,1], A, P)$ has no equilibrium point.

In this section, we have proved the existence theorems of equilibria for generalized games with compact and infinite dimensional strategy spaces, an infinite number of agents, and nontotal-nontransitive constraint and $U$-majorized preference correspondences which may not have open graphs or open lower (upper) sections.

Since we, also, know that in the infinite settings, the set of feasible allocations generally is not compact in any topology of the commodity spaces. It is necessary to consider the existence of equilibria for generalized games in which the strategy spaces are not compact. This is done by strengthening the assumptions on the preference or constraint correspondences which enables one to remove altogether the compactness (or paracompactness) assumptions on the strategy spaces in the following section. 
5. Maximal elements and equilibria for $U$-majorized condensing mappings. In this section, we consider the existence theorems of equilibria for non-compact qualitative games and non-compact generalized games in which the strategy spaces are not compact.

We first have the following existence theorem of equilibria of generalized game in which the constraint mappings are $\Psi$-condensing.

THEOREM 5.1. Let $\varphi=\left(X_{i} ; A_{i}, B_{i} ; P_{i}\right)_{i \in I}$ be a generalized game and $X=\prod_{i \in I} X_{i}$ such that for each $i \in I$,

(i) for each $i \in I, X_{i}$ is a nonempty closed convex subset of a locally convex Hausdorff topological vector space $E_{i}$;

(ii) for each $i \in I, A_{i}: X \longrightarrow 2^{X_{i}}$ is such that for each $x \in X, A_{i}(x)$ is nonempty and $\operatorname{co} A_{i}(x) \subset \overline{B_{i}}(x)$;

(iii) for each $i \in I$, the set $E^{i}=\left\{x \in X:\left(A_{i} \cap P_{i}\right)(x) \neq \varnothing\right\}$ is open and paracompact in $X$;

(iv) for each $i \in I, A_{i} \cap P_{i}$ is U-majorized on $E^{i}$;

(v) the mapping $B: X \longrightarrow 2^{X}$ defined by $B(x)=\prod_{i \in I} \overline{B_{i}}(x)$ for each $x \in X$ is $\Psi$ condensing, where $\Psi: 2^{\prod_{i \in I} E_{i}} \longrightarrow C$ is a measure of non-compactness.

Then $G$ has an equilibrium point in $X$, i.e., there exists a point $\hat{x}=\left(\hat{x}_{i}\right)_{i \in I} \in X$ such that for each $i \in I, \hat{x}_{i} \in \overline{B_{i}}(\hat{x})$ and $A_{i}(\hat{x}) \cap P_{i}(\hat{x})=\varnothing$.

Proof. Since the mapping $B: X \longrightarrow 2^{X}$ is $\Psi$-condensing, by Lemma 2.1, there exists a nonempty compact and convex subset $K$ in $X$ such that $B: K \longrightarrow 2^{K}$.

Now, we follow the proof of Theorem 4.1. Note that if $E^{i}=\varnothing$ for all $i \in I$, then the conclusion follows by Fan-Glicksberg fixed point theorem again (e.g., see Fan [8] or Glicksberg [14]).

Let $I_{0}=\left\{i \in I: E^{i} \neq \varnothing\right\}$. Without loss of generality, we may assume that $I_{0} \neq \varnothing$.

CASE 1. For each $i \in I_{0}$, by (iv) and Theorem 3.1, there exists a mapping $\psi_{i}: E^{i} \longrightarrow 2^{X_{i}}$ which is upper semicontinuous with closed and convex values and $A_{i}(x) \cap P_{i}(x) \subset$ $\psi_{i}(x)$ for each $x \in E^{i}$. Since $\overline{B_{i}}: X \longrightarrow 2^{X_{i}}$ is upper semicontinuous with closed and convex values, the mapping $\psi_{i} \cap \overline{B_{i}}: X \rightarrow 2^{X_{i}}$ is, also, upper semicontinuous with nonempty closed and convex values by Lemma 2.3 on $E^{i}$. Define a correspondence $\phi_{i}: X \longrightarrow 2^{X_{i}}$ by

$$
\phi_{i}(x)= \begin{cases}\overline{B_{i}}(x), & \text { if } x \notin E^{i}, \\ \left(\psi_{i} \cap \overline{B_{i}}\right)(x), & \text { if } x \in E^{i} .\end{cases}
$$

Then Lemma 2.2 implies that $\phi_{i}$ is upper semicontinuous with nonempty closed and convex values.

CASE 2. For $i \in I \backslash I_{0}$, we define a correspondence $\phi: X \longrightarrow 2^{X_{i}}$ by $\phi_{i}:=\overline{B_{i}}(x)$ for each $x \in X$. Then $\phi$ is upper semicontinuous with nonempty compact and convex values.

Finally, we define a correspondence $\Psi: X \longrightarrow 2^{X}$ by $\Psi(x):=\prod_{i \in I} \phi_{i}(x)$ for each $x \in X$. Then $\Psi$ is, also, upper semicontinuous with nonempty compact and convex values. Since $\Psi(x) \subset B(x)$ for each $x \in X$ and $B$ is self-mapping in $K$, the restriction of $\Psi$ on $K$ is also self-map. Now, Fan-Glicksberg fixed point theorem implies that there exists a point $x \in K$ such that $x \in \Psi(x)$. If there exists $i \in I_{0}$ such that $x \in E^{i}$, then 
$\pi_{i}(x) \in \phi_{i}(x)=\overline{B_{i}}(x) \cap \psi_{i}(x) \subset \psi_{i}(x)$ which contradicts that $\psi_{i}$ is $U$-majorized on $E^{i}$. Therefore, $x \notin E^{i}$ for all $i \in I_{0}$, i.e., there exists an $i^{\prime} \notin I_{0}$ such that $x \in E^{i^{\prime}}$. By the definition of $\Psi$, we must have $\pi_{i}(x) \in \overline{B_{i}}(x)$ and $A_{i}(x) \cap P_{i}(x)=\varnothing$ for all $i \in I$.

Finally, we have the following maximal element theorem for $U$-majorized condensing correspondence.

THEOREM 5.2. Let $X$ be a nonempty closed and convex subset of a Hausdorff locally convex topological vector space E. Let $P: X \rightarrow 2^{X}$ be $U$-majorized (i.e., $\cup_{I_{X}}$-majorized) and $\Psi$-condensing, where $\Psi: 2^{\prod_{i \in I} E_{i}} \longrightarrow C$ is a measure of non-compactness. Then there exists a point $x \in X$ such that $P(x)=\varnothing$.

Proof. By Lemma 2.1, there exists a nonempty compact and convex subset $K$ of $X$ such that $P: K \rightarrow 2^{K}$. Then it is the same as that of Theorem 4.1 except for the application of Fan-Glicksberg fixed point theorem to $K$.

For the existence of equilibria of abstract economies (or generalized games) in which preferences are not $U$-majorized in topological vector spaces or locally convex topological vector spaces, we refer to Borglin and Keiding [2], Chang [4], Ding et al. [6], Gale and Mas-Colell [13], Shafer and Sonnenschein [28], Tan and Yuan [30], Tan and Yuan [29], Tarafdar [31], Tian [32], Toussiant [33], Tulcea [17], Yannelis and Prabhakar [36] and the references wherein. We, also, remark that an existence result of equilibria for abstract economy which is related to Theorem 4.1 was proven by Kim in [18] under the different assumptions, e.g., $P(x)$ is nonempty and convex for all $x \in X$ or $P(x)=\varnothing$ for all $x \in X$.

ACKNOWLEDGEMENT. The authors express grateful thanks to anonymous referee for his/her careful reading and comments which lead to the present version of this paper.

\section{REFERENCES}

[1] T. C. Bergstrom, Maximal elements of acyclic preference relations on compact sets, J. Econom. Theory 10 (1975), 403-404. Zbl 325.04001.

[2] A. Borglin and H. Keiding, Existence of equilibrium actions and of equilibrium: a note on the "new" existence theorems, J. Math. Econom. 3 (1976), no. 3, 313-316. MR 562451. Zbl 349.90157.

[3] Ju. G. Borisovic, B. D. Gelman, A. D. Myskis, and V. V. Obuhovskii, Topological methods in the fixed-point theory of multi-valued maps, Uspekhi Mat. Nauk 35 (1980), no. 1(211), 59-126,255 (Russian). MR 81e:55004. Zbl 464.55003.

[4] S. Y. Chang, On the Nash equilibrium, Soochow J. Math. 16 (1990), no. 2, 241-248. MR 91m:90028. Zbl 721.90017.

[5] G. Debreu, A social equilibrium existence theorem, Proc. Nat. Acad. Sci. U.S.A. 38 (1952), 886-893. MR 14,301c. Zbl 047.38804.

[6] X. P. Ding, W. K. Kim, and K. K. Tan, Equilibria of noncompact generalized games with $L^{*}$-majorized preference correspondences, J. Math. Anal. Appl. 164 (1992), no. 2, 508-517. MR 93d:90014. Zbl 765.90092.

[7] J. Dugundji, Topology, Allyn and Bacon, Inc., Boston, 1966. MR 33\#1824. Zbl 144.21501.

[8] K. Fan, Fixed-point and minimax theorems in locally convex topological linear spaces, Proc. Nat. Acad. Sci. U.S.A. 38 (1952), 121-126. MR 13,858d. Zbl 047.35103. 
[9] P. M. Fitzpatrick and W. V. Petryshyn, Fixed point theorems for multivalued noncompact acyclic mappings, Pacific J. Math. 54 (1974), no. 2, 17-23. MR 538973. Zbl 312.47047.

[10] M. Florenzano, L'équilibre economique général transitif et intransitif: Problems d'éxistance, CNRS, Paris, 1981 (French).

[11] M. Furi and A. Vignoli, A fixed point theorem in complete metric spaces, Boll. Un. Mat. Ital. 4 (1969), no. 2, 505-509. MR 41\#1034. Zbl 183.51404.

[12] D. Gale and A. Mas Colell, An equilibrium existence theorem for a general model without ordered preferences, J. Math. Econom. 2 (1975), no. 1, 9-15. MR 522542. Zbl 324.90010.

[13] _ On the role of complete, translative preferences in equlibrium theory, Equilibrium and Disequilibrium in Economics Theory (G.Schwödiauer, ed.), pp. 7-14, Reidel, Dordrecht, 1978.

[14] I. L. Glicksberg, A further generalization of the Kakutani fixed theorem, with application to Nash equilibrium points, Proc. Amer. Math. Soc. 3 (1952), 170-174. MR 13,764g. Zbl 046.12103.

[15] W. Hildenbrand, Core and equilibria of a large economy, Princeton Studies in Mathematical Economics, vol. 5, Princeton University Press, Princeton, 1974, With an appendix to Chapter 2 by K. Hildenbrand. MR 52 9991. Zbl 351.90012.

[16] C. J. Himmelberg, Fixed points of compact multifunctions, J. Math. Anal. Appl. 38 (1972), 205-207. MR 46 2505. Zbl 225.54049.

[17] C. Ionescu Tulcea, On the approximation of upper semi-continuous correspondences and the equilibriums of generalized games, J. Math. Anal. Appl. 136 (1988), no. 1, 267289. MR 90b:90156. Zbl 685.90100.

[18] W. K. Kim, A new equilibrium existence theorem, Econom. Lett. 39 (1992), no. 4, 387-390. MR 93i:90131. Zbl 769.90085.

[19] E. Klein and A. C. Thompson, Theory of correspondences, Canadian Mathematical Society Series of Monographs and Advanced Texts, John Wiley \& Sons, Inc., New York, 1984, Including applications to mathematical economics. MR 86a:90012. Zbl 556.28012.

[20] C. Kuratowski, Sur les espaces completes, Fund. Math. 15 (1920), 301-309.

[21] A. S. Markus, I. T. Gohberg, and L. S. Goldenstein, Investigations of some properties of bounded linear operators with their q-norms, Uch. Zap. Kishinevsk. In-Ta. 29 (1957), 29-36 (Russian).

[22] P. Massatt, Some properties of condensing maps, Ann. Mat. Pura Appl. 4 (1980), no. 125, 101-115. MR 82j:54099. Zbl 446.54041.

[23] G. Mehta, Maximal elements of condensing preference maps, Appl. Math. Lett. 3 (1990), no. 2, 69-71. MR 91b:47129. Zbl 717.47020.

[24] R. D. Nussbaum, The fixed point index for local condensing maps, Ann. Mat. Pura Appl. 4 (1971), no. 89, 217-258. MR 47 903. Zbl 226.47031.

[25] W. V. Petryshyn and P. M. Fitzpatrick, Fixed-point theorems for multivalued noncompact inward maps, J. Math. Anal. Appl. 46 (1974), 756-767. MR 5014232. Zbl 287.47038.

[26] S. Reich, Fixed points in locally convex spaces, Math. Z. 125 (1972), 17-31. MR 466110. Zbl 224.47031.

[27] B. N. Sadovskii, Limit-compact and condensing operators, Uspehi Mat. Nauk 27 (1972), no. 1(163), 81-146 (Russian). MR 55 1161. Zbl 243.47033.

[28] W. Shafer and H. Sonnenschein, Equilibrium in abstract economies without ordered preferences, J. Math. Econom. 2 (1975), no. 3, 345-348. MR 53 2315. Zbl 312.90062.

[29] K. K. Tan and X. Z. Yuan, Lower semicontinuity of multivalued mappings and equilibrium points, World Congress of Nonlinear Analysis '92 (Berlin), vol. I-IV, Walter de Gruyter, 1996, pp. 1849-1860. CMP 96 12. Zbl 863.47031. 
[30] K. K. Tan and Z. Z. Yuan, A minimax inequality with applications to existence of equilibrium points, Bull. Austral. Math. Soc. 47 (1993), no. 3, 483-503. MR 94g:47075. Zbl 803.47059.

[31] E. Tarafdar, A fixed point theorem and equilibrium point of an abstract economy, J. Math. Econom. 20 (1991), no. 2, 211-218. MR 91k:90037. Zbl 718.90014.

[32] G. Q. Tian, Equilibrium in abstract economies with a noncompact infinite-dimensional strategy space, an infinite number of agents and without ordered preferences, Econom. Lett. 33 (1990), no. 3, 203-206. MR 91e:90027.

[33] S. Toussaint, On the existence of equilibria in economies with infinitely many commodities and without ordered preferences, J. Econom. Theory 33 (1984), no. 1, 98-115. MR 85k:90048. Zbl 543.90016.

[34] M. Walker, On the existence of maximal elements, J. Econom. Theory 16 (1977), no. 2, 470-474. MR 57 10680. Zbl 421.54016.

[35] N. C. Yannelis, Maximal elements over noncompact subsets of linear topological spaces, Econom. Lett. 17 (1985), no. 1-2, 133-136. MR 86i:90011.

[36] N. C. Yannelis and N. D. Prabhakar, Existence of maximal elements and equilibria in linear topological spaces, J. Math. Econom. 12 (1983), no. 3, 233-245. MR 87h:90061a. Zbl 536.90019.

YuAN: DePARTMENT OF MATHEMATICS, STATISTICS AND COMPUTING SCIENCE, DALHOUSIE UNIVersity, HalifaX, Nova Scotia B3H 3J5, CANADA; AND Department of Mathematics, The UNIVERSITY OF QUEENSLAND, BRISBANE 4072, AUSTRALIA

TARAFDAR: DEPARTMENT OF MATHEMATICS, THE UNIVERSITY OF QUEENSLAND, BRISBANE 4072, Australia 


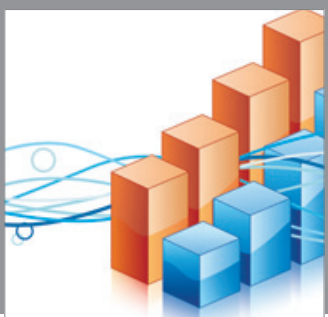

Advances in

Operations Research

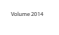

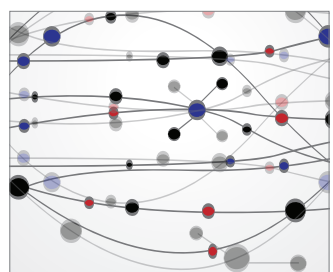

\section{The Scientific} World Journal
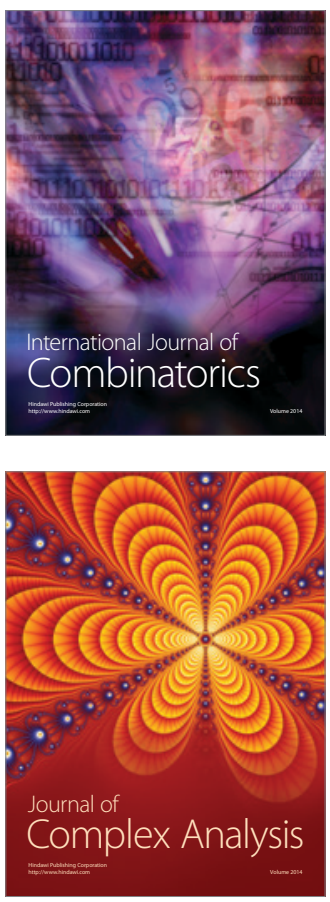

International Journal of

Mathematics and

Mathematical

Sciences
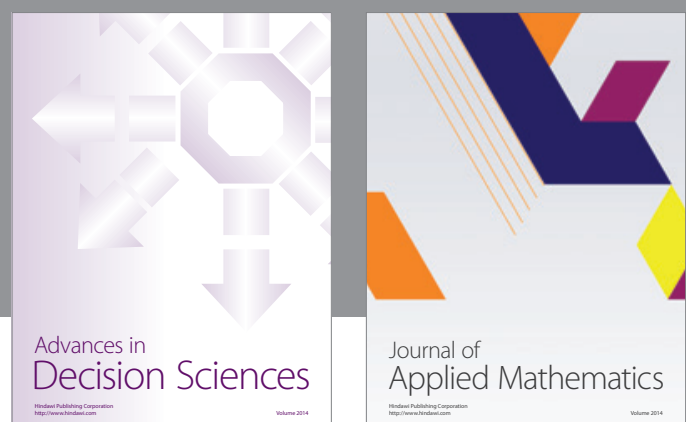

Journal of

Applied Mathematics
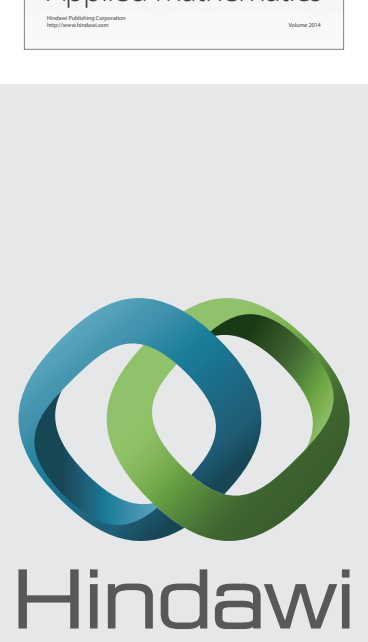

Submit your manuscripts at http://www.hindawi.com
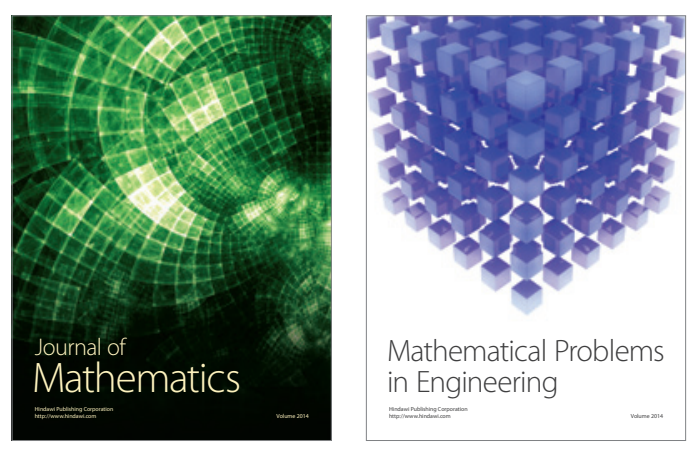

Mathematical Problems in Engineering
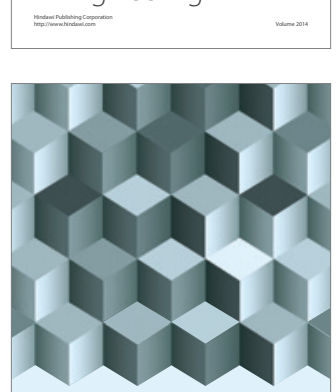

Journal of

Function Spaces
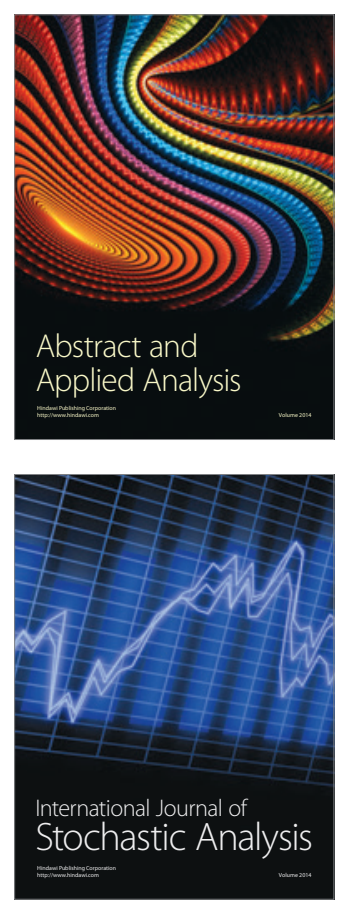

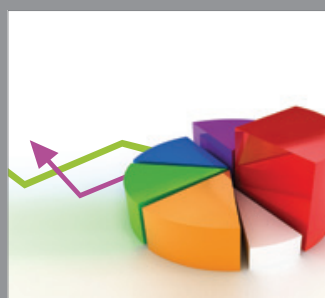

ournal of

Probability and Statistics

Promensencen
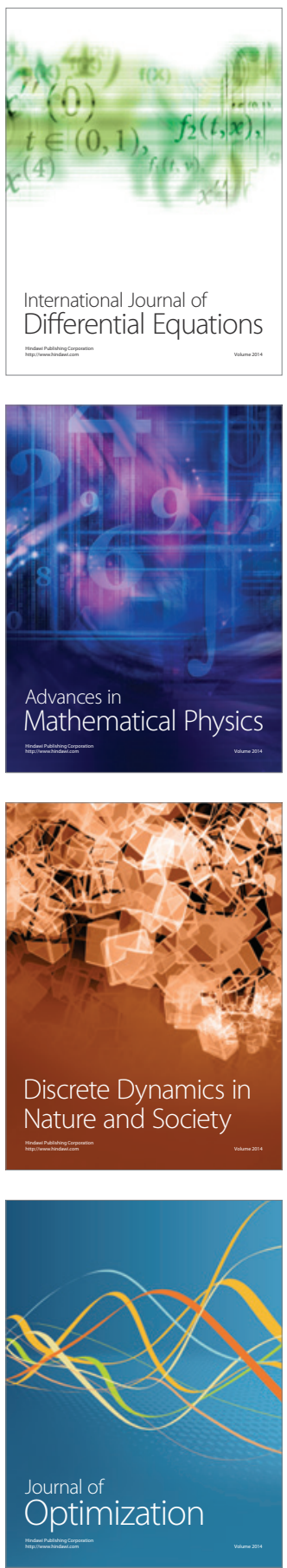